Este trabajo ha sido seleccionado para su publicación por:

Don Gorka J. Palazio Arko

Don Antonio Bautista García-Vera

Don José María de Moya Anegón

Don Javier García Vilumbrales

Doña Clara Eugenia Núñez Romero-Balmas

\title{
El porfolio electrónico como recurso educativo y su impacto en la búsqueda de trabajo
}

\section{Extracto:}

Nuestro artículo pretende evidenciar que el porfolio electrónico puede ser una excelente herramienta de aprendizaje y de evaluación, así como un currículum vitae multimedia, el cual se puede enviar fácilmente a futuros empleadores. El porfolio electrónico recoge información sobre competencias y experiencias personales y profesionales tanto formales como informales. Igualmente, presenta trabajos, certificados y otro tipo de pruebas elegidos por el individuo para ilustrar sus habilidades y competencias y evidenciar sus logros. En este artículo, se explicarán los diferentes tipos y partes del porfolio electrónico y se demostrará que el porfolio electrónico enseña una serie de estrategias que facilitan el proceso de aprendizaje y adquisición de habilidades necesarias en el mundo laboral. Además, se presentarán ejemplos reales de porfolios electrónicos en su versión estadounidense y española que han sido desarrollados por estudiantes universitarios en ambos países. Finalmente, se analizarán los resultados de una encuesta a directores de recursos humanos y se demostrará que el porfolio electrónico puede considerarse una herramienta de búsqueda de trabajo muy útil y eficaz que cuenta con una significativa aprobación en el mundo de la empresa.

\footnotetext{
${ }^{1}$ M. Ciesielkiewicz, profesora del centro universitario Villanueva, adscrito a la Universidad Complutense de Madrid.
} 


\section{The}

ePortiolio as an educational resourse and its impact on job search

\section{Abstract:}

This paper intends to make evident that the ePortfolio is an effective and cuttingedge learning and evaluation tool, as well as a multimedia curriculum vitae that can be sent out to future employers. It can be used to present, document, reflect on, and promote students' skills, credentials, certificates and diverse formal and informal experiences in a structured, wellorganized and marketable way.

Different types and parts of ePortfolio will be explained in this paper and it will be demonstrated that ePortfolio teaches a ranges of strategies that facilitates learning, as well as the acquisition of skills needed in a workplace. Besides, real examples of ePortfolio, both american and spanish version, developed by students in both countries will be presented. Finally, the results of a survey carried out among fifty two human resources directors will be analyzed. This paper will demonstrate that the electronic portfolio can be considered a very useful and effective job search tool that can count with a significant approval in the business world.

Keywords: electronic portfolio, ePortfolio, information and communication technologies (ICT), human resources, educational resource, teaching tool, education, e-learning, evaluation.

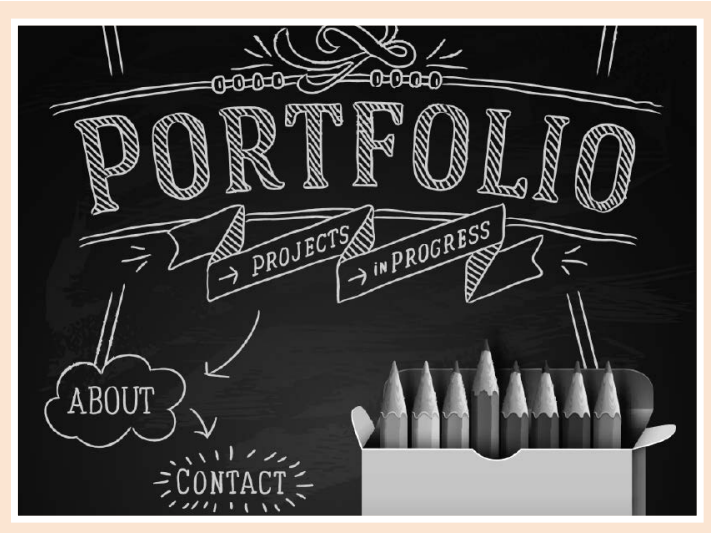

\section{INTRODUCCIÓN}

Hoy en día existe una amplia oferta de recursos tecnológicos para la enseñanza. Las nuevas tecnologías constituyen una inmensa fuente de material didáctico que puede facilitar el proceso de aprendizaje de cualquier materia. Sin duda alguna, son una herramienta a la que los estudiantes actuales dan preferencia y a la que acuden con mayor frecuencia tanto para buscar información sobre cualquier tema como para adquirir conocimientos propios de su campo de especialización. Asimismo, el Plan Bolonia recomienda la incorporación de nuevas tecnologías en la enseñanza para fomentar la formación continua y la autonomía del alumno.

Por otro lado, no hay que caer en una actitud de excesiva confianza en cuanto a la aplicación de las nuevas tecnologías en el aula. Estas, per se, no suponen una mejora ni en la impartición de contenidos didácticos por parte del profesor ni en la adquisición de conocimientos por parte del alumno. Como cualquier instrumento de trabajo hay que saber utilizarlo adecuadamente para que cumpla con los objetivos didácticos que deseamos obtener. Uno de estos recursos, que es relativamente nuevo y reconocido internacionalmente, pero poco utilizado en España, es el porfolio electrónico (denominado comúnmente por el término anglosajón ePortfolio).

El objetivo principal de nuestro trabajo consiste en demostrar que el porfolio electrónico puede ser un excelente instrumento de aprendizaje y evaluación durante la etapa académica, así como una herramienta de búsqueda de trabajo una vez concluida dicha etapa. En este trabajo se explicarán sus diferentes tipos y partes y se demostrará que el porfolio electrónico enseña una serie de estrategias que facilitan el proceso de aprendizaje y adquisición de habilidades necesarias en el mundo laboral. Además, se presentarán ejemplos reales de porfolios electrónicos en su versión estadounidense y es- 
pañola que han sido desarrollados por estudiantes universitarios en ambos países. Finalmente, se analizarán los resultados de una encuesta a directores de recursos humanos y se demostrará que el porfolio electrónico puede considerarse una herramienta de búsqueda de trabajo que cuenta con una significativa aprobación en el mundo de la empresa.

El porfolio electrónico está reconocido en el ámbito internacional por numerosos países miembros del Consejo de Europa y ha despertado un gran interés en EE. UU., Canadá y Nueva Zelanda, dando lugar a varios proyectos relacionados con el tema, así como en Australia, donde se ha financiado e implementado como proyecto gubernamental. Desgraciadamente, este recurso tan útil e innovador es muy poco conocido en España a pesar de ser objeto de un proyecto europeo denominado «ePortfolio for All», cuyo patrocinio corre a cargo del Consejo de Europa.

Por otro lado, según la United Nations Educational, Scientific and Cultural Organization (UNESCO) y la Organización para la Cooperación y el Desarrollo Económicos (OCDE), la educación continua es uno de los factores de «la prosperidad económica y estabilidad social» (Watson, 2003, pág. 1). La UNESCO también pone énfasis en la importancia y necesidad de desarrollar habilidades y conocimiento, así como formación permanente a lo largo de la vida. Delors (1996, pág. 100) la define como «un imperativo para la democracia», mientras que DEST (2007, pág. 10) la clasifica como «empleabilidad en el futuro». La educación continua es una realidad y una exigencia del mundo laboral que el alumno tiene que asumir y a la cual tiene que adaptarse. Sin duda alguna, el porfolio electrónico promueve tanto la inserción en el mercado laboral como la educación permanente formal e informal.

En ocasiones, desde distintos estamentos empresariales se menciona que lo que demandan las empresas está lejos de lo que ofrecen las universidades y las escuelas de negocios. Es más, se suele afirmar que la diferencia entre lo que se enseña en el mundo académico y la práctica que es demandada por el mundo empresarial provoca que nuestros jóvenes tengan dificultades para acceder al mercado laboral. Creemos que el uso del porfolio electrónico puede ayudar a conciliar ambos mundos desde el primer momento en que un joven tiene contacto con la empresa, que es durante el proceso de selección, concretamente con los Departamentos de Recursos Humanos. El hecho de que un aspirante a obtener un empleo utilice como carta de presentación una herramienta que ha ido construyendo a lo largo de su vida académica con el soporte de sus profesores puede contribuir a que la distancia aparente entre ambos mundos se reduzca y sea un primer ejemplo de que lo aprendido en la universidad tiene una aplicación directa en la empresa.
(...) se suele afirmar que la diferencia entre lo que se enseña en el mundo académico y la práctica que es demandada por el mundo empresarial provoca que nuestros jóvenes tengan dificultades para acceder al mercado laboral. Creemos que el uso del porfolio electrónico puede ayudar a conciliar ambos mundos

\section{MARCO TEÓRICO}

\subsection{Definición del porfolio electrónico}

Definir lo que es un porfolio electrónico resulta una tarea compleja y confusa puesto que es un término muy amplio y es utilizado en muchos contextos y de distintas maneras en diferentes países. Además, el porfolio electrónico puede tener múltiples usos y aplicaciones. Varias definiciones del porfolio electrónico reconocen que es una colección de trabajos, documentos e ítems seleccionados por el propietario del porfolio electrónico. Asimismo, el Centre for Recording Achievement (CRA) ha establecido ciertas características y definiciones que exponemos a continuación:

- Un repositorio de documentos.

- Un medio para acceder a información personal que normalmente está guardada en diferentes bases de datos.

- Un medio de presentarse a uno mismo a los demás, así como evidenciar sus habilidades, cualidades y logros.

- Un medio de recoger y seleccionar pruebas de evaluación.

- Una herramienta de orientación para facilitar el repaso y la elección.

- Un medio para compartir información y colaborar.

- Un medio para fomentar el sentimiento de identidad personal (Ward y Grant, 2007). 
En resumen, es un documento electrónico en el que una persona puede presentar información sobre su formación académica y experiencias profesionales. Recoge las experiencias y competencias obtenidas tanto dentro como fuera del ámbito educativo. El porfolio electrónico puede usarse como un currículum vitae electrónico y multimedia, puesto que contiene información sobre experiencias de aprendizaje y de trabajo y ejemplos reales del dominio de distintas competencias.

El papel del porfolio electrónico para representar la identidad digital ha ido ganando atención e interés [European Institute for E-Learning (ElfEL), 2008 a]. Podemos afirmar que el porfolio electrónico tiene grandes ventajas en comparación con el porfolio en papel. Es fácil de actualizar e imprimir. Es económico, multimedia y fácilmente transportable. Toda la información del porfolio electrónico puede ser descargada en una base de datos que puede ser usada por los profesores y las instituciones para hacer estudios del proceso de aprendizaje de los estudiantes. Los estudiantes pueden tener acceso a su porfolio electrónico a través de la red para ver la evolución de su aprendizaje. El porfolio electrónico se puede personalizar de acuerdo con las exigencias profesionales. La estructura digital permite la coexistencia de muchos tipos de documentos y evita la sobrecarga de gráficos. Una versión digital permite tener un porfolio multimedia. En el dossier, el estudiante puede recoger no solo documentos de su trabajo en papel, sino también en vídeo y audio. El porfolio digital es fácil de enviar por correo electrónico como documento adjunto o un enlace y es más rápido de rellenar y modificar que un porfolio en papel. Además, permite crear porfolios para estudiantes universitarios con vistas a su incorporación en el mercado de trabajo. Por último, proporciona a las instituciones una herramienta que permite controlar el proceso de aprendizaje de los estudiantes.

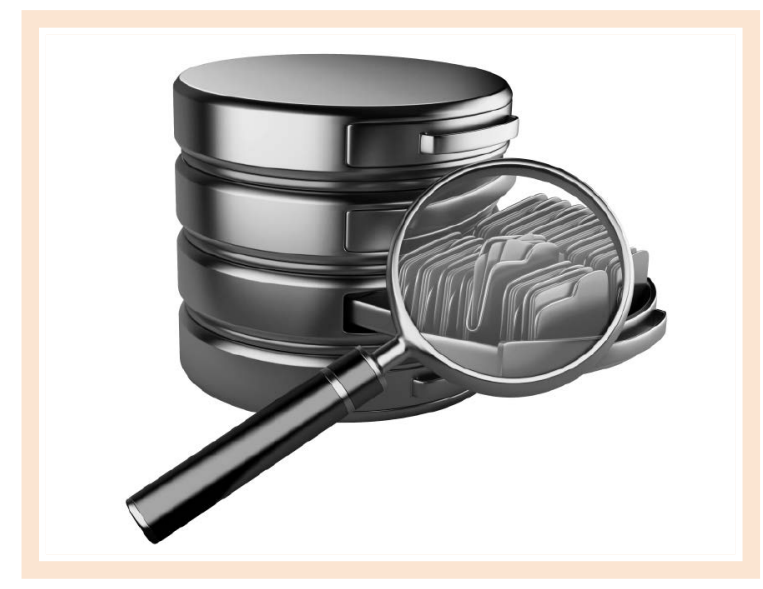

\subsection{Tipos de porfolios electrónicos}

El hecho de que existan numerosos objetivos y usos de los porfolios electrónicos hace la tarea de clasificarlos bastante complicada. Diferentes autores han propuesto distintas tipologías, sin embargo, realmente son los propios usuarios quienes los definen, les dan forma y establecen los objetivos de su creación y uso acorde a sus necesidades concretas.

IMS Global Learning Consortium, la asociación que desarrolla estándares y apoya las mejores prácticas en el área de tecnologías educativas, ha identificado seis grandes tipos de porfolios electrónicos, entre los cuales se encuentran los siguientes (IMS, 2005):

\section{- Porfolio electrónico de evaluación (assessment ePort-}

folio). Se usa para demostrar el logro de ciertos objetivos especificados por algún tipo de autoridad. Frecuentemente, se utilizan rúbricas con el fin de calificar los porfolios electrónicos de evaluación. Diferentes universidades, facultades, departamentos y escuelas los usan para obtener la acreditación de conocimientos adquiridos por parte de sus alumnos y para las prácticas profesionales, por ejemplo, en el campo de la educación, el arte o la enfermería.

\section{- Porfolio electrónico de presentación (presentation} ePortfolio). Se usa para demostrar los resultados de aprendizaje, éxito académico y profesional de manera convincente a través de las pruebas aportadas. Contiene instrucciones sobre su contenido y cómo deberían interpretarse. Los porfolios electrónicos de presentación se usan a menudo para enseñar cualificaciones, capacidades o títulos profesionales con el propósito de solicitar trabajo o de demostrar logros para pedir promoción. 
- Porfolio electrónico de aprendizaje (learning ePortfolio). Se utiliza para documentar, guiar y promover el aprendizaje a lo largo del tiempo. Se caracteriza por un elevado componente de reflexión y se puede usar para promover la metacognición, la planificación del aprendizaje, o para integrar diversas experiencias de aprendizaje. Se desarrollan más frecuentemente en contextos curriculares formales. Por ejemplo, en el caso de los alumnos universitarios que tienen que entregar trabajos de una asignatura y compartir sus observaciones y reflexiones sobre su proceso de aprendizaje y adquisición de nuevos conocimientos.

- Porfolio electrónico de desarrollo personal (personal development ePortfolio). La planificación de desarrollo personal se define como un proceso estructurado y sostenido realizado por un individuo para reflexionar sobre su aprendizaje, rendimiento y desempeño, así como para planificar su desarrollo a nivel personal, educativo y profesional. Por lo tanto, el porfolio electrónico de desarrollo personal incluye tanto un porfolio electrónico de aprendizaje como un porfolio electrónico profesional.

- Porfolio electrónico de varios propietarios (multiple-owner ePortfolio). Se utiliza con el objetivo de dar acceso a su contenido a varios usuarios. Puede combinar elementos de otros tipos de porfolios electrónicos anteriormente mencionados, sin embargo, lo más frecuente es que tenga la forma de un porfolio electrónico de presentación, como, por ejemplo, una página web o un blog. Puede también tener aspecto de un porfolio electrónico de aprendizaje en el que se muestran los resultados de un trabajo colaborativo y evidencias de adquisición de competencias. Asimismo, el porfolio electrónico de varios propietarios puede ser un porfolio institucional en el que se presentan el trabajo, las actividades y el desarrollo de proyectos de una organización.

- Porfolio electrónico de trabajo (working ePortfolio). Combina elementos de todos los tipos de porfolios electrónicos anteriormente mencionados. Recoge visiones y reflexiones de sus usuarios. Puede parecerse al porfolio electrónico de evaluación, exposición, aprendizaje o de desarrollo. El acceso al porfolio electrónico de trabajo lo tiene un número reducido de personas, mientras que otras solo pueden ver y leer el contenido sin poder modificarlo.

\section{El porfolio electrónico puede usarse como un currículum vitae electrónico y multimedia, puesto que contiene información sobre experiencias de aprendizaje y de trabajo y ejemplos reales del dominio de distintas competencias}

\subsection{Partes del porfolio electrónico}

Como se ha mencionado anteriormente, los usos y las aplicaciones del porfolio electrónico varían según su propietario y sus necesidades concretas. Igualmente, las partes y la información compartida cambian en base al mismo criterio. Según Smith y Tilemma (2003), los porfolios electrónicos suelen incluir la siguiente información sobre su propietario:

- Información personal.

- Historial académico.

- Reconocimiento-premios y certificados.

- Comentarios de reflexión.

- Trabajos del curso-tareas del curso, proyectos.

- Comentarios del profesor.

- Comentarios del empleador anterior.

- Objetivos y planes.

- Valores personales e intereses.

- Exposiciones y trabajos.

- Actividades personales-voluntariado y desarrollo personal.

A continuación vamos a presentar ejemplos reales de porfolios electrónicos de alumnos de tres universidades: Virginia Commonwealth University, Messiah College en Pensilvania y Centro Universitario Villanueva adscrito a la Universidad Complutense de Madrid. Estos porfolios electrónicos han sido desarrollados por estudiantes del último año de carrera, matriculados en la asignatura de Senior Seminar o Senior Portfolio, en el caso de las dos universidades estadounidenses, y de segundo año de Magisterio, matriculados en la asignatura de 
El porfolio electrónico como recurso educativo y su impacto en la búsqueda de trabajo / M. Ciesielkiewicz

Tecnologías de la Información y la Comunicación, en el caso de la universidad española. Cabe destacar la dificultad para encontrar universidades españolas que hayan implementado el porfolio electrónico en su currículo, al contrario de lo que sucede en los centros educativos de EE. UU. Esta es una muestra evidente de que el porfolio electrónico sigue siendo poco conocido en España.

El porfolio electrónico es un proyecto de las asignaturas anteriormente mencionadas que los alumnos tienen que entregar obligatoriamente y que a la vez les sirve como un currículum vitae electrónico y multimedia que pueden enviar a sus futuros empleadores. Cada alumno tiene que ir subiendo a su porfolio electrónico diferentes trabajos asignados a lo largo de un cuatrimestre y que luego convierte en su currículum vitae multimedia añadiendo más documentación pertinente al mundo laboral. Las plataformas que usamos al principio para facilitar la creación de porfolios electrónicos a nuestros estudiantes fueron Blackboard y Sakai. Las dos son muy fáciles de usar, sin embargo, tienen una gran desventaja: los estudiantes dejan de tener acceso a su porfolio electrónico después de graduarse. Por lo tanto, hemos decidido utilizar Google Site que es aún más fácil de usar y permite a nuestros estudiantes tener acceso y modificar su porfolio electrónico a lo largo de su vida.

Veamos algunos ejemplos.

\section{Figura 1. Porfolio electrónico de una alumna de Senior Seminar}
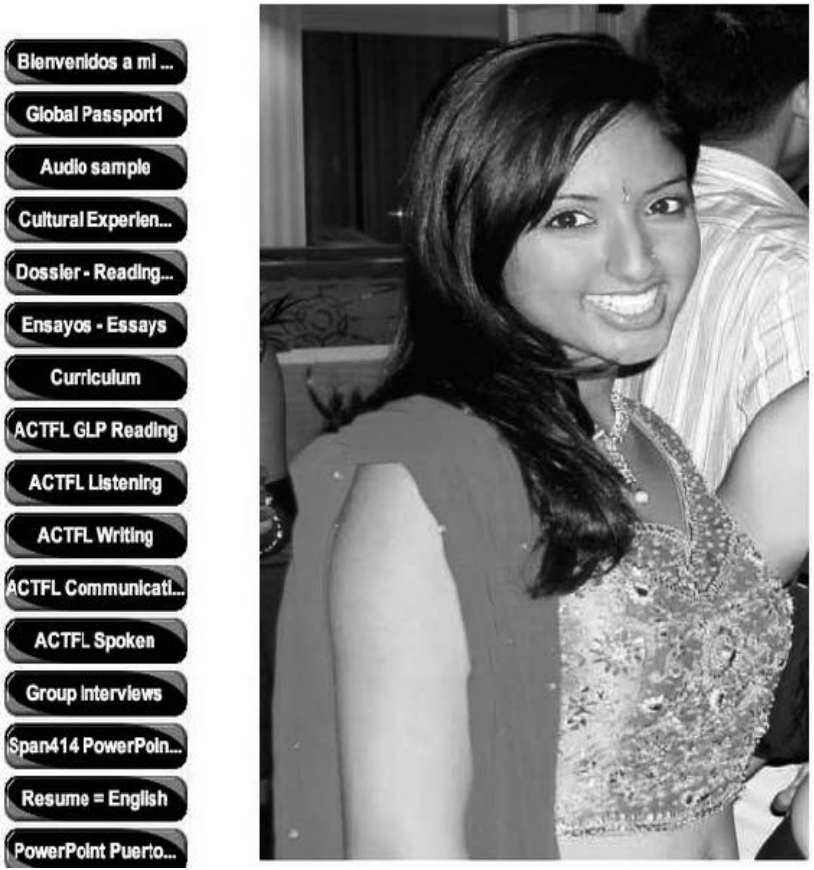

Fuente: VCU Global Language Portfolio Samples. 
M. Ciesielkiewicz / El porfolio electrónico como recurso educativo y su impacto en la búsqueda de trabajo

\section{Figura 2. Porfolio electrónico de un alumno de Tecnologías de la Información y la Comunicación}

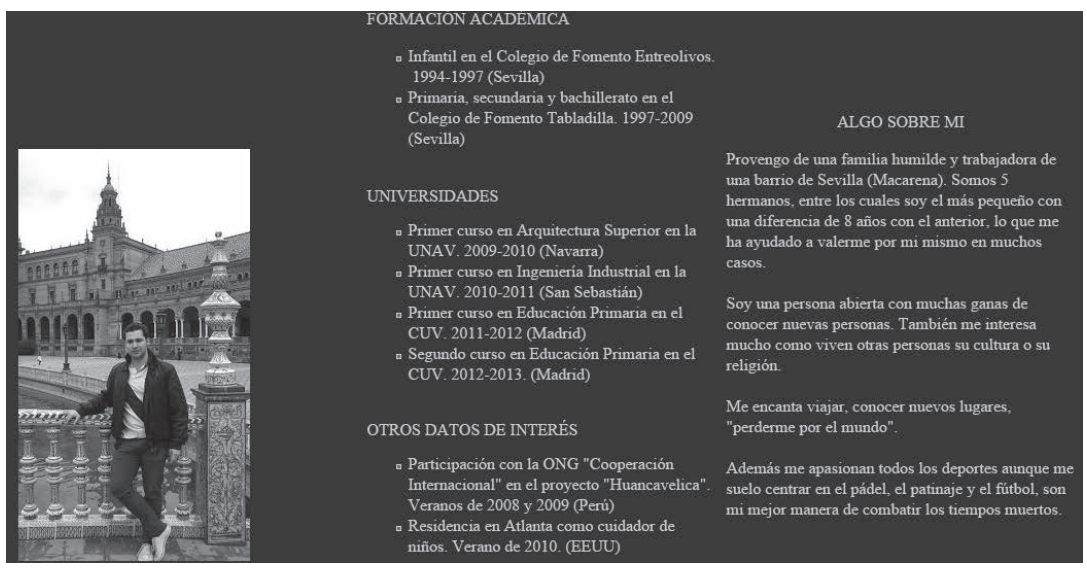

Fuente: elaboración propia.

\section{Figura 3. Biografía. Estrategias de aprendizaje}

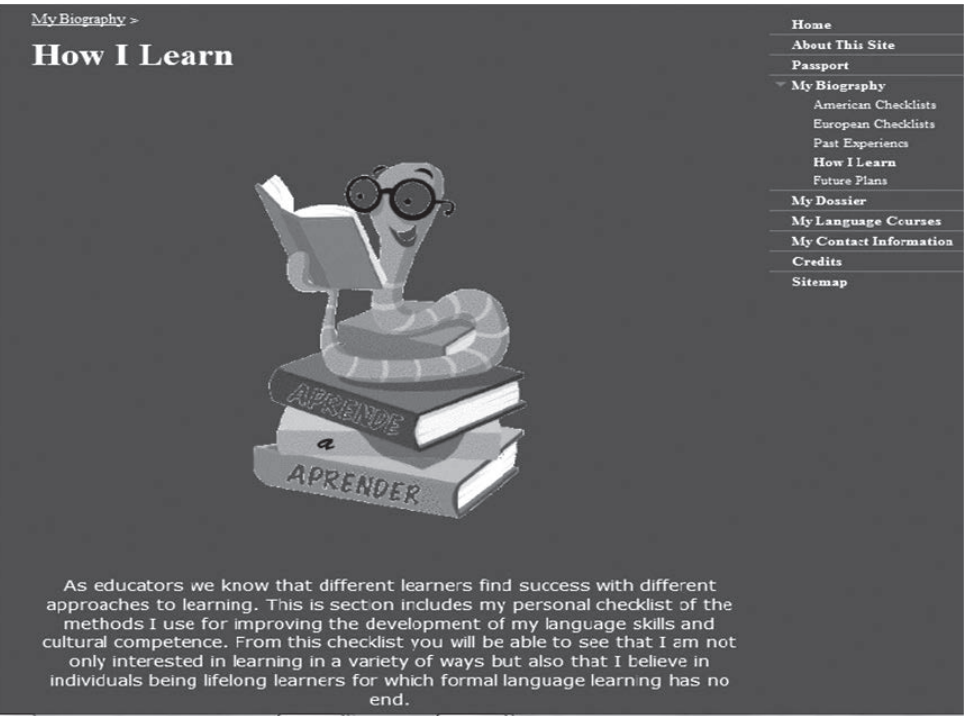

Fuente: elaboración propia. 
El porfolio electrónico como recurso educativo y su impacto en la búsqueda de trabajo / M. Ciesielkiewicz

Figura 4. Ejemplos de trabajos y proyectos
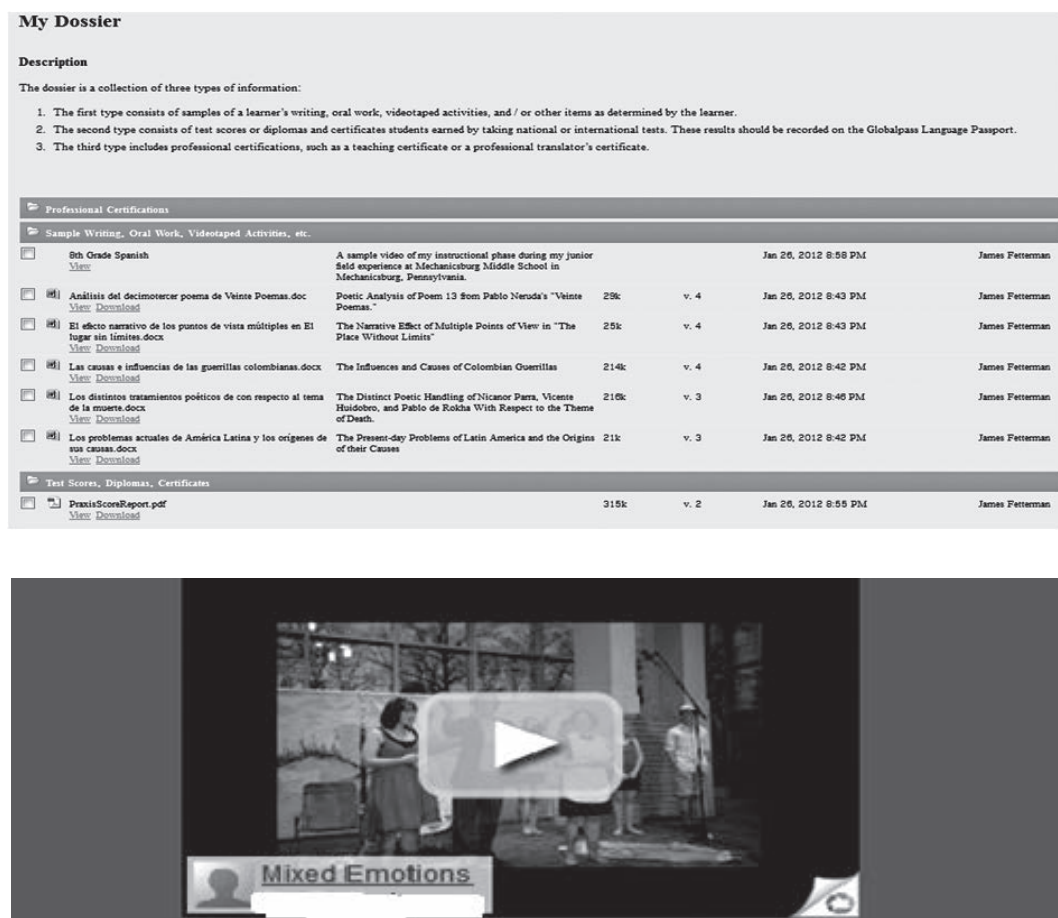

Messiah college-Grantham, PA

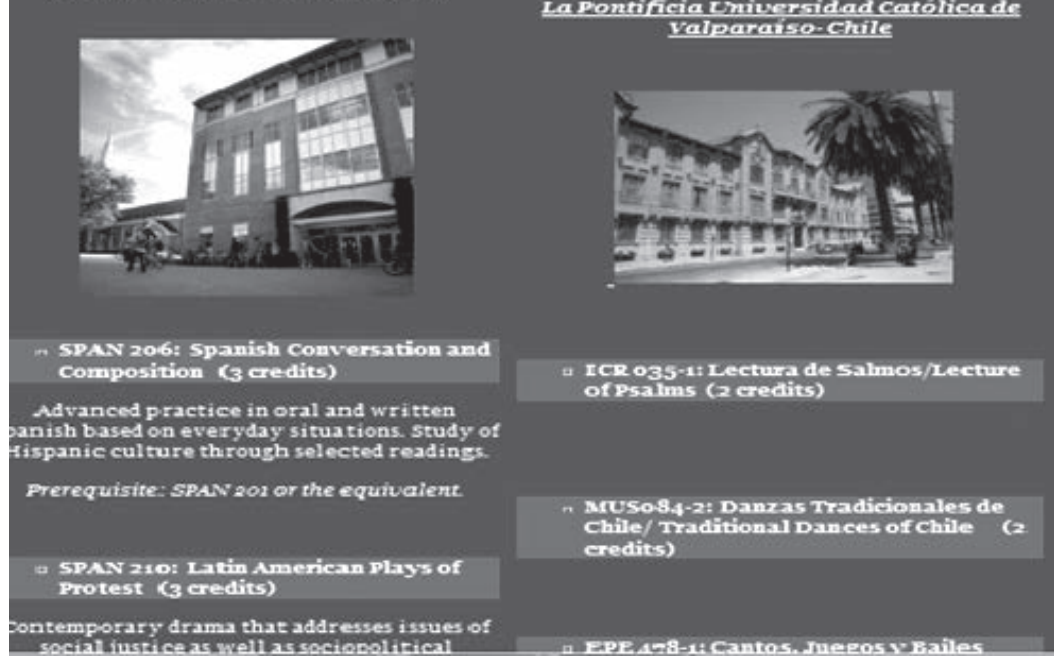

La Pontificia rmiversidad Católiea de Kalparaiso-chite

Fuente: elaboración propia. 


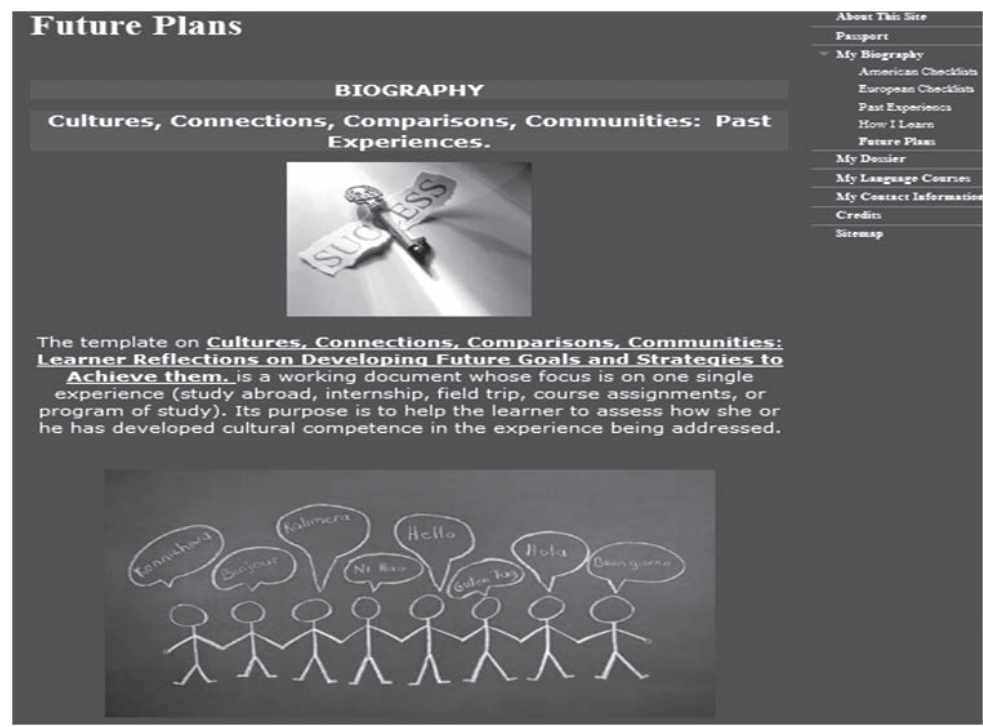

\subsection{El porfolio electrónico como herramienta de aprendizaje y evaluación}

El porfolio electrónico es un excelente ejemplo de cómo se pueden promover, enseñar y aprender eficazmente estrategias de aprendizaje y metacognición que facilitan este proceso y que pueden servir a los alumnos toda la vida. Les enseña a evaluarse a sí mismos y a identificar sus competencias y capacidades actuales. El estudiante tiene que planificar lo que va a aprender, reflexionar sobre su proceso de aprendizaje dentro y fuera del sistema escolar, así como describir sus experiencias formales e informales, remuneradas o no remuneradas, pero que pueden ser beneficiosas para sus estudios y carrera profesional. Calderón y Hernández (2006, pág. 2) opinan que el porfolio electrónico ayuda a los alumnos a reflexionar sobre sus experiencias y «crear una conexión entre la teoría y la práctica». Diamond (2006) también está de acuerdo en que la reflexión es un aspecto esencial de los porfolios electrónicos. Igualmente, el porfolio electrónico anima e inspira a los estudiantes a que empleen un amplio abanico de estrategias y técnicas de estudio con el propósito de llevar a cabo sus objetivos individuales.

Paris (1994) afirma que los estudiantes que no están motivados y no tienen voluntad de aprender encuentran difícil asimilar y desarrollar estrategias de aprendizaje. El porfolio electrónico documenta y demuestra al estudiante sus logros y su éxito en el proceso de aprendizaje y le motiva para seguir estudiando, esforzarse y luchar contra sus inhibiciones. De igual modo, le ayuda a mantener el mismo nivel de entusiasmo respecto a su desarrollo personal y profesional. Por estas razones, el porfolio electrónico ayuda de una manera explícita a desarrollar las estrategias afectivas, como, por ejemplo, saber animarse y reducir la ansiedad.

Entonces, ¿por qué implementar el porfolio electrónico como herramienta de aprendizaje y evaluación en 
el currículo? Merece la pena hacerlo dado que el porfolio electrónico enseña a los alumnos a:

- Establecer objetivos a corto y largo plazo.

- Identificar estrategias para alcanzar sus objetivos.

- Organizar mejor el material que quieren aprender.

- Evaluar su propio conocimiento.

- Poner en práctica la autorreflexión y la autoevaluación.

- Observar su progreso y saber motivarse a sí mismos.

- Determinar las fortalezas, debilidades y preferencias.

- Responsabilizarse de su aprendizaje.

- Evidenciar su éxito.

- Desarrollar estrategias personales como parte de la formación continua para toda la vida.

Oxford (1990) asevera que las estrategias de aprendizaje promueven la autonomía del discente, ya que el alumno no va a tener al profesor a su lado todo el tiempo y no siempre va a poder contar con su ayuda. Wenden y Rubin (1987) confirman también que la autonomía y la autogestión promueven el aprendizaje dentro y fuera del aula. De igual manera, los estudiantes que conocen y emplean estrategias eficaces sabrán seguir estudiando cuando ya no puedan contar con el apoyo y asesoramiento del profesor.

Sutherland (2005) opina que el porfolio electrónico no solo debería enseñarse como un trabajo 0 proyecto de una asignatura, sino que debería convertirse en un instrumento de desarrollo personal. Debería ser una herramienta de myLearning (mi aprendizaje) en lugar de solo e-learning (aprendizaje virtual).

\section{El porfolio electrónico documenta y demuestra al estudiante sus logros y su éxito en el proceso de aprendizaje y le motiva para seguir estudiando, esforzarse y luchar contra sus inhibiciones}

\section{MÉTODO}

\subsection{Tipo y diseño de investigación}

Para la realización de este trabajo, se ha llevado a cabo una investigación cuyo objetivo radica en un análisis del uso actual del porfolio electrónico por parte de los directores de recursos humanos, así como de la brecha que puede existir entre el mundo educativo y la empresa.

En otras palabras, se trata de un estudio de índole descriptiva, ya que pretende ahondar en la utilidad y aplicabilidad de una herramienta didáctica que puede servir como recurso de educación permanente y herramienta de búsqueda de trabajo.

\subsection{Población y muestra}

Para conocer si realmente los directores de recursos humanos iban a tener en cuenta el porfolio electrónico a la hora de seleccionar candidatos y si lo consideraban útil, hemos realizado tres preguntas de respuesta abierta y cinco de tipo test, cerradas y de creación propia, con el objetivo de plantear una serie de cuestiones relacionadas con el uso del porfolio electrónico en el mundo de la empresa a una «muestra» de 52 directores de recursos humanos. 52 directores de recursos humanos de los siguientes países: España, Portugal, México, Colombia, Brasil, Argentina, Italia, Holanda y Suecia.

Aunque la muestra seleccionada de los países es bastante amplia, reconocemos también que la mayoría de los encuestados provienen de España, por tanto, quizá este estudio podría ampliarse más en este sentido en futuras investigaciones.

En cualquier caso, y en términos generales, el estudio de la presente muestra puede servir para obtener una primera aproximación orientativa acerca del objeto de estudio de este trabajo. 


\subsection{Procedimiento}

El procedimiento seguido en el trabajo parte de una serie de aspectos de índole práctica como son el planteamiento del problema, la situación actual del uso de porfolios electrónicos en el ambiente educativo a nivel internacional y su aplicación y transcendencia en el mundo de la empresa. Asimismo, una vez presentados los datos obtenidos de las encuestas realizadas, se pasará a una propuesta de medidas que podrían llevarse a cabo para dar a conocer más el porfolio electrónico tanto en la educación como en la empresa.

\subsection{Técnica de recolección de datos}

Como se puede observar en este apartado del trabajo, el instrumento elegido para la recolección de datos ha sido una encuesta. En efecto, todas las preguntas planteadas habian sido elaboradas previamente, tres seguían la estructura de respuesta abierta y cinco de respuesta cerrada, es decir, sin ofrecer la posibilidad de responder algo diferente a las contestaciones planteadas en la misma encuesta. Las tres primeras preguntaban sobre el país donde trabajaba el encuestado, el número de empleados de su empresa en dicho país y si habían visto un currículum vitae multimedia alguna vez. Las siguientes cuestiones respondían a aspectos más relacionados con las actitudes de los directores de recursos humanos respecto al uso y la percepción del porfolio electrónico como herramienta de selección de candidatos para un puesto de trabajo.

\subsection{Técnica de análisis de datos}

A la luz de los datos obtenidos a partir de las encuestas, se puede afirmar que la técnica predominantemente utilizada a la hora de analizar dicha información es de tipo cuantitativo.

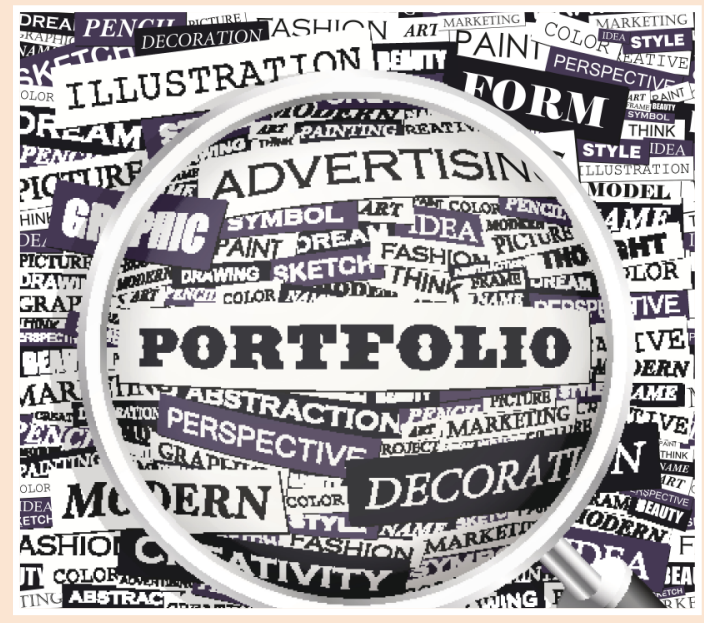

\subsection{Presentación y discusión de los resultados}

\subsubsection{Resultados del análisis}

Pregunta 1. La primera cuestión por la que se les interroga a los encuestados es el país en el que trabajan.

\section{Figura 6. Origen de empresas encuestadas}

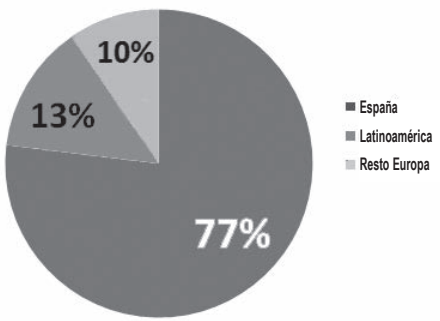

Fuente: elaboración propia

Como se puede observar en el gráfico anterior, el resultado a la primera pregunta es el siguiente: un $77 \%$ de los directores de recursos humanos provienen de España, un $10 \%$ del resto de Europa y un $13 \%$ de Latinoamérica. 
El porfolio electrónico como recurso educativo y su impacto en la búsqueda de trabajo / M. Ciesielkiewicz

Pregunta 2. La segunda cuestión está planteada para conocer el tamaño de la empresa; en concreto, el número de empleados. El resultado de la pregunta se muestra en la siguiente gráfica.

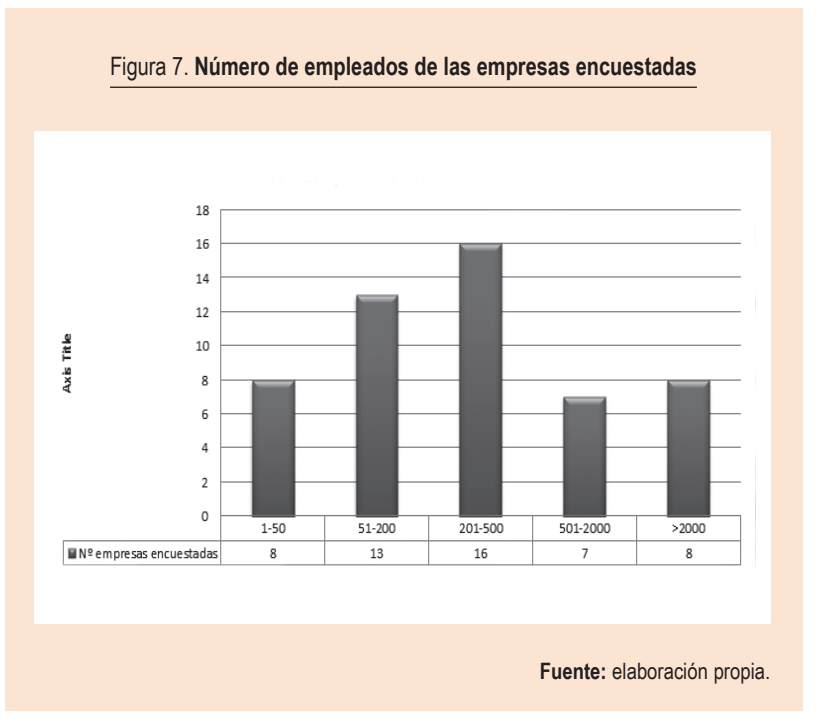

Como se puede observar en la tabla, las empresas que han participado en la encuesta se han clasificado en cinco categorías:

1. Empresas de entre 1 a 50 empleados, que en términos porcentuales representan un $15 \%$ de los encuestados (8 empresas).

2. Empresas de 51 a 200, que representan un $25 \%$ de los participantes en la encuesta (13 empresas).

3. Entre 201 a 500 empleados, que constituyen un $31 \%$ de los encuestados ( 16 empresas).

4. Entre 501 a 2.000 empleados, que en la encuesta suponen el $14 \%$ del total (7 empresas).

5. Empresas de más de 2.000 empleados, que representan un $15 \%$ de todos los encuestados (8 empresas).

Pregunta 3. La tercera cuestión es: ¿había visto un currículum vitae multimedia alguna vez?

\section{Figura 8. Conocimiento previo del porfolio electrónico}

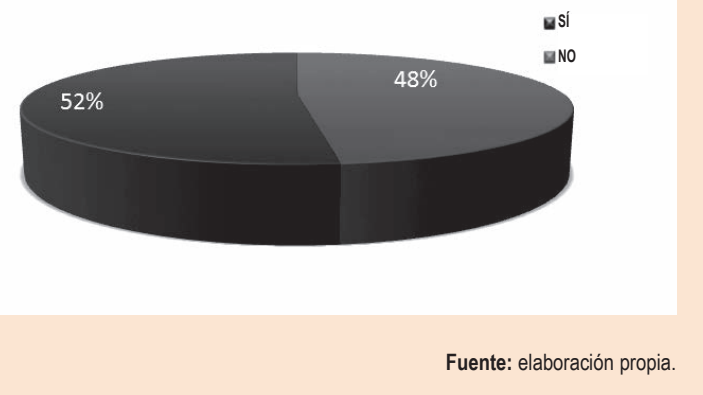

El $48 \%$ responde afirmativamente, un total de 25 personas. Por otra parte, 27 personas, un $52 \%$, veían por primera vez un currículum vitae multimedia. Observamos que el currículum vitae multimedia no es ampliamente conocido entre los directores de recursos humanos, siendo ligeramente superior, un $4 \%$, el número de personas que desconocen la existencia del mismo.

Hasta aquí se han llevado a cabo las preguntas abiertas. A partir de ahora las siguientes cuestiones irán encaminadas al uso del porfolio electrónico como herramienta de búsqueda de

empleo. Las siguientes cinco preguntas tienen forma de afirmaciones. Se pidió a los encuestados que indicaran cuán verdaderas/ciertas eran dichas enunciaciones siguiendo la escala de 1 a 7 , donde 1 significa totalmente en desacuerdo y 7 totalmente de acuerdo (1. Totalmente en desacuerdo; 2. En desacuerdo; 3 . Algo en desacuerdo; 4 . Ni de acuerdo ni en desacuerdo; 5 . Algo de acuerdo; 6. De acuerdo; 7. Totalmente de acuerdo). 
M. Ciesielkiewicz / El porfolio electrónico como recurso educativo y su impacto en la búsqueda de trabajo

Pregunta 4. La cuarta cuestión de la encuesta está planteada para saber si el encuestado utilizaría el currículum vitae multimedia para seleccionar candidatos para un puesto de trabajo.

Figura 9. Currículum vitae multimedia para seleccionar candidatos

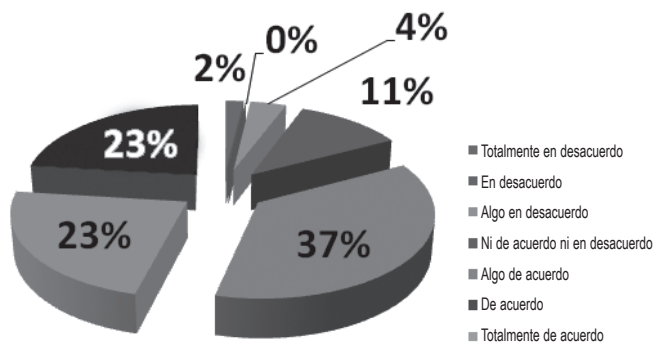

Fuente: elaboración propia.
Como podemos ver, la gran mayoría de los directores de recursos humanos que contestaron a esta pregunta, un $83 \%$ del total, utilizarían el currículum vitae multimedia para seleccionar candidatos para un puesto de trabajo; en concreto, un $37 \%$ de los encuestados estaban algo de acuerdo, un $23 \%$ de acuerdo y otro $23 \%$ declaraban estar totalmente de acuerdo. El $11 \%$ del total no estaba ni de acuerdo ni en desacuerdo, un $4 \%$ lo consideraban poco probable y solo un $2 \%$ no lo utilizarían.

Pregunta 5. La quinta cuestión versa sobre si el encuestado haría el esfuerzo de revisar el currículum vitae multimedia de todos los candidatos para seleccionar a los mejores para un puesto de trabajo.

\section{Figura 10. Currículum vitae multimedia de todos}

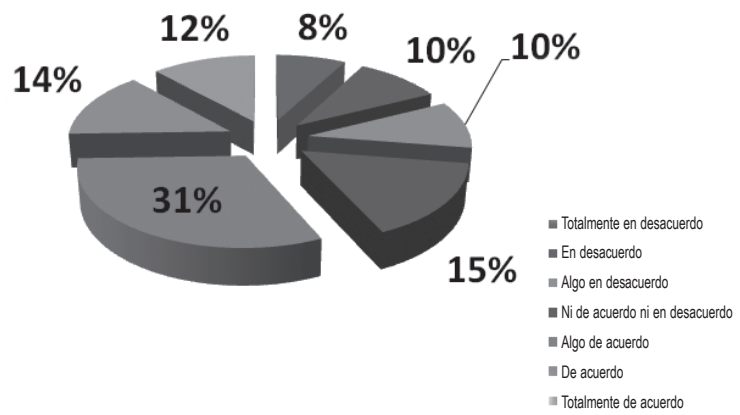

Fuente: elaboración propia.
Analizando esta pregunta podemos observar que un $26 \%$ de los directores de recursos humanos ciertamente revisarian el currículum vitae multimedia de todos los candidatos para un puesto de trabajo; entre ellos un $14 \%$ está de acuerdo y un $12 \%$ totalmente de acuerdo. El $31 \%$ del total están algo de acuerdo, por lo tanto, probablemente lo revisarían. El $15 \%$ de los encuestados se mantiene neutral, es decir, no están ni de acuerdo ni en desacuerdo, un $10 \%$ está algo en desacuerdo, otro $10 \%$ en desacuerdo y un $8 \%$ lo descarta por completo. 
El porfolio electrónico como recurso educativo y su impacto en la búsqueda de trabajo / M. Ciesielkiewicz

Pregunta 6. En la sexta cuestión planteada a los encuestados se les pregunta si harían el esfuerzo de revisar el currículum vitae multimedia de los tres mejores candidatos para un puesto de trabajo.

\section{Figura 11. Currículum vitae multimedia de los tres mejores candidatos}

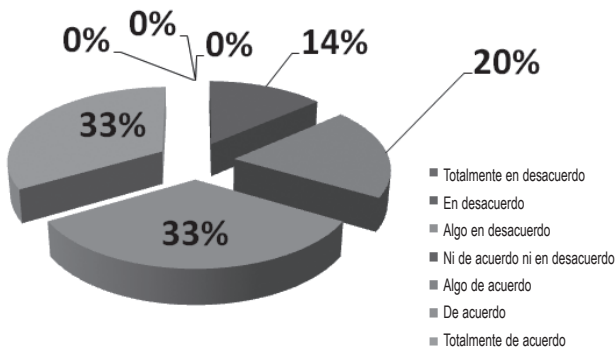

Fuente: elaboración propia
Como se puede observar, el $66 \%$ de los encuestados afirma que revisaría los currículum vitae multimedia de los tres mejores candidatos; la mitad de ellos está de acuerdo y la otra mitad totalmente de acuerdo. Les siguen los que están algo de acuerdo con un $20 \%$ del total. Todos ellos suman un $86 \%$ de todos los encuestados que revisarían el currículum vitae multimedia de los tres mejores candidatos para un puesto de trabajo. El $14 \%$ del total no está ni de acuerdo ni en desacuerdo. Puede llamar la atención que nadie está en desacuerdo con la cuestión planteada.

Pregunta 7. En la siguiente cuestión se pregunta si el encuestado haría el esfuerzo de revisar el currículum vitae multimedia del mejor candidato para un puesto de trabajo.

\section{Figura 12. Currículum vitae multimedia del mejor candidato}

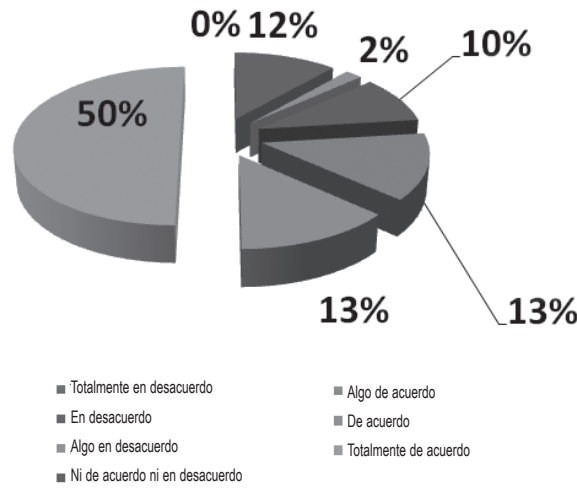

Fuente: elaboración propia
Según se puede observar en la gráfica, la gran mayoría de directores de recursos humanos que han contestado a esta pregunta asegura que revisaría el currículum vitae multimedia del mejor candidato para un puesto de trabajo. Esta es la respuesta mayoritaria que en términos porcentuales representa un $63 \%$ de los encuestados; entre ellos un $50 \%$ estaban totalmente de acuerdo y un $13 \%$ de acuerdo. Si se añaden los que están algo de acuerdo, se llegaría a la conclusión de que el $76 \%$ de todos los encuestados revisarían el currículum vitae multimedia del mejor candidato. Por otro lado, un $2 \%$ está algo en desacuerdo y un $12 \%$ en desacuerdo, que suman un $14 \%$ del total.

Ninguno de los encuestados está totalmente en desacuerdo. Finalmente, un $10 \%$ no está ni de acuerdo ni en desacuerdo. 
M. Ciesielkiewicz / El porfolio electrónico como recurso educativo y su impacto en la búsqueda de trabajo

Pregunta 8. En la última cuestión se pregunta por la opinión de los encuestados acerca del porfolio electrónico como currículum vitae multimedia, en concreto, si les parece una herramienta útil para obtener información sobre los candidatos para un puesto de trabajo.

\section{Figura 13. Valoración sobre el currículum vitae multimedia como herramienta para seleccionar candidatos}

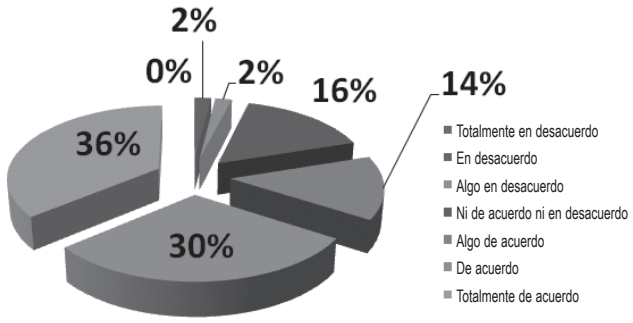

Fuente: elaboración propia.
La gran mayoría de los directores de recursos humanos que han participado en la encuesta, un $80 \%$, consideran el currículum vitae multimedia una herramienta útil para obtener información más amplia y detallada sobre los candidatos para un puesto de trabajo; entre ellos un $36 \%$ están totalmente de acuerdo, un $30 \%$ están de acuerdo y un $14 \%$ algo de acuerdo. Los que no están ni de acuerdo ni en desacuerdo suman un $16 \%$ del total. A estas cifras les siguen de lejos los que afirman estar algo en desacuerdo, que son tan solo un $2 \%$, y los que están en desacuerdo, otro $2 \%$.

Cabe añadir que no se ha analizado la relación entre el país de origen y el tamaño de la empresa y su impacto en el uso del porfolio electrónico. Se ha decidido excluirlo para estudiar una serie de vertientes más detalladas en investigaciones futuras.

\subsubsection{Discusión de resultados}

Una vez presentados los resultados de la encuesta, nos preguntamos: ¿merece la pena incluir el porfolio electrónico en el currículo de la formación universitaria? La respuesta es sí, todos los recursos que puedan ayudar a los jóvenes a conseguir trabajo y que puedan reducir la brecha existente entre el mundo laboral y el universitario deben ser bienvenidos e implantados con entusiasmo. Los resultados de la encuesta lo demuestran claramente. Como hemos observado, la gran mayoría de los directores de recursos humanos que han participado en la encuesta, esto es, un $83 \%$, utilizarían el currículum vitae multimedia para seleccionar candidatos para un puesto de trabajo. El $86 \%$ de todos los encuestados revisarían el currículum vitae multimedia de los tres mejores candidatos para seleccionar al mejor. Cabe mencionar aquí que un $26 \%$ de los encuestados aseguran revisar el currículum vitae multimedia de todos los encuestados y un $31 \%$ esta algo de acuerdo. Sin embargo, los resultados de esta pregunta no sorprenden, ya que se requeriría mucho tiempo para llevar a cabo dicha tarea y más aún en empresas grandes. Por otro lado, el $76 \%$ de todos los encuestados afirman revisar el currículum vitae multimedia del mejor candidato. Finalmente, la gran mayoría de los directores de recursos humanos que han participado en la encuesta, en concreto un $80 \%$, consideran el currículum vitae multimedia una herramienta útil para obtener información más amplia y detallada sobre los candidatos para un puesto de trabajo.

En resumen, el porfolio electrónico es un excelente instrumento tanto de evaluación como de aprendizaje, así como una herramienta de búsqueda de trabajo. El currículum vitae electrónico y multimedia puede servir para documentar e ilustrar habilidades, experiencias y logros. Es un archivo del proceso de aprendizaje personal que ayuda al individuo a evaluar sus objetivos a corto y largo plazo, su desarrollo personal, educativo y profesional, así como a reflexionar sobre sus experiencias. Nuestra investigación demuestra que además de ser un recurso educativo puede servir como herramienta de búsqueda de empleo que cuenta con una significativa aprobación en el mundo de la empresa. 


\section{CONCLUSIONES}

La adopción del porfolio electrónico en los países miembros de la Unión Europea, EE.UU., Canadá, Nueva Zelanda y Australia demuestra un gran interés y reconocimiento por este instrumento a escala internacional. Desgraciadamente, en España el porfolio electrónico es poco conocido y utilizado. Faltan pautas prácticas, material didáctico, guías y talleres para profesores sobre la incorporación del porfolio electrónico al currículo. Por lo tanto, es necesario y urgente dar a conocer este recurso tan innovador, eficaz y útil tanto para el mundo educativo como laboral. Además de usarse como instrumento de evaluación por parte de diversas instituciones educativas en diferentes partes del mundo, el porfolio electrónico sirve también como una excelente herramienta didáctica, así como un currículum vitae electrónico y multimedia.

Como se ha mencionado anteriormente, el porfolio electrónico constituye una herramienta que recoge información sobre competencias y experiencias personales y profesionales tanto formales como informales. Igualmente, presenta trabajos, certificados y otro tipo de pruebas elegidos por el individuo para ilustrar sus habilidades y competencias y evidenciar sus logros. Por consiguiente, puede servir perfectamente como un currículum vitae multimedia y una herramienta de búsqueda de empleo.

Cabe añadir que la formación continua como parte de la educación permanente formal, informal o en el lugar de trabajo es imprescindible en estos tiempos de cambio tecnológico rápido y de globalización. El alumno de hoy tiene que ser independiente, autónomo y asumir la responsabilidad de su proceso de aprendizaje, lo cual es esencial para el éxito profesional. Además, le ayuda a determinar propósitos personales respecto al aprendizaje y establecer objetivos reales, así como a identificar las estrategias a corto y largo plazo necesarias para llevar a cabo sus metas individuales. Por lo tanto, se le enseña a desarrollar estrategias personales como parte de la formación continua para toda la vida. En definitiva, el porfolio electrónico es una herramienta muy eficaz de educación permanente que promueve la autonomía y la responsabilidad del alumno.

El porfolio electrónico, evidentemente, ayuda a acercar el mundo universitario al laboral dado que facilita la inserción en el mercado de trabajo. Aparte de los
(...) el porfolio electrónico puede ayudar a los empleadores a identificar el nivel de preparación y aptitud de los candidatos para un puesto de trabajo, así como a sacar mejor provecho de sus capacidades y competencias

grandes beneficios para los alumnos anteriormente mencionados, el porfolio electrónico puede ayudar a los empleadores a identificar el nivel de preparación y aptitud de los candidatos para un puesto de trabajo, así como a sacar mejor provecho de sus capacidades y competencias. Les puede ahorrar tiempo y esfuerzo en la selección de candidatos, ya que gracias al porfolio electrónico los Departamentos de Recursos Humanos pueden establecer unas expectativas más realistas para las entrevistas de trabajo basadas en un entendimiento más claro de la formación, capacidades y experiencia profesional de los candidatos. Además, les puede ayudar a determinar qué tipo de curso, taller, instrucción o experiencia son necesarias para alcanzar un nivel más avanzado de competencias profesionales 0 a diseñar un proceso individualizado de aprendizaje más efectivo tras su incorporación a la empresa.

En resumen, el porfolio electrónico ofrece grandes ventajas tanto para el mundo educativo como laboral. Nuestro trabajo pone de manifiesto que es necesario e inminente darlo a conocer en España, especialmente en el nivel de educación superior como apoyo a la mejora de la empleabilidad de los estudiantes y para facilitar su inserción en el mercado de trabajo. Además, podría realizarse una investigación cualitativa y cuantitativa más completa y detallada en torno a la incidencia del uso del porfolio electrónico para el desarrollo de las competencias académicas y profesionales, dados los indicios descubiertos. 
M. Ciesielkiewicz / El porfolio electrónico como recurso educativo y su impacto en la búsqueda de trabajo

\section{BIBLIOGRAFÍA}

Australian ePortfolio Project (AeP) [2008]: Australian ePortfolio project. Disponible en: http://www.ePortfoliopractice.qut.edu.au [Consulta: 15 de enero de 2015].

Calderón M. y Hernández, F. [2006]: Implementing an ePortfolio system through nonacademic areas, paper presented at VirtualEduca2006. Disponible en: http:// inm.ccadet.unam.mx/virtualeduca2006/ pdf/106-MMC.pdf [Consulta: 10 de febrero de 2015].

Candy, P. [1995]: Developing lifelong learners through undergraduate education, paper presented at the Teaching and Learning Forum. Disponible en: http:// Isn.curtin.edu.au/t|f/t|f1995/candy.html [Consulta: 25 de marzo de 2015].

Centre for Recording Achievement (CRA) [2008]: About CRA. Disponible en: http:// www.recordingachievement.org/ [Consulta: 15 de enero de 2015].

Chea, T.: Elite colleges transform online higher education. Recuperado el 25 de marzo de 2015 de: http://finance.yahoo. com/news/elite-colleges-transform-online-higher-124855202.html.

Cole, D. J.; Ryan, C. W. y Kick, F. [1995]: Portfolios across the curriculum and beyond, Thousand Oaks (CA), Corwin Press.

Cummins, P. y Davesne, C. [2009]: «Using electronic portfolios for second language assessment», The Modern Language Journal, 93, págs. 848-861.

Declaración de Bolonia [1999]: Disponible en: http://www.eees.es/es/documentacion [Consulta: 15 de enero de 2015].

Delors, J. [1996]: Learning: the treasure within, París, United Nations Educational Scientific and Cultural Organization.

Department of Education, Science and Training (DEST) [2007]. Employability skills. Disponible en: http://www.dest.gov.au/sectors/training_skills/policy_issues_reviews/ key_issues/es [Consulta: 3 de septiembre de 2015].
Diamond, S. [2006]. Diversity is essential. Disponible en: http:// ncepr.org/ncepr/drupal/blog/121 [Consulta: 4 de marzo de 2015].

Eflickles, A. [2011]: «Interactions of metacognition with motivation and affect in self-regulated learning: the MASRL Model», Educational Psychologist, 46 (1), págs. 6-25. Doi: 10.1080/00461520.2011.538645.

Gibson, D. [2006]: «ePortfolio decisions and dilemmas», en A. Jafari y C. Kaufman (eds.), Handbook of research on ePortfolios, Hershey (PA), Idea Group, págs. 135-145.

Global Language Portfolio Samples. Imagen tomada del sitio Global Language Portfolio. Disponible en: http://glp.elenes.com/ sampleswelcome.html [Consulta: 8 de septiembre de 2010]).

Johnson, R.; Mims-Cox, J. S. y Doyle-Nichols, A. [2006]: Developing portfolios in education: a guide to reflection, inquiry, and assessment, London, Sage.

IMS Global Learning Consortium Inc. [2005]. IMS ePortfolio best practice and implementation guide. Disponible en: http://www. imsglobal.org/ep/epv1p0/imsep_bestv1p0.html [Consulta: 15 de enero de 2015].

Oxford, R. [1990]: Language learning strategies, Boston (MA), Heinle and Heinle.

Paris, S. y Ayres, L. [1994]: Becoming reflective students and teachers with portfolios and authentic assessment, Washington, DC, American Psychological Association.

Sitzmann, T. y Ely, K. [2010]: «Sometimes you need a reminder: the effects of prompting self-regulation on regulatory processes, learning and attrition», Journal of Applied Psychology, 95 (1), págs. 132-144. Doi: 10.1037/a0018080.

Smith, K. y H. Tilemma, H. [2003]. "Clarifying different types of portfolio use», Assessment and Evaluation in Higher Education, 28 (6), págs. 625- 648.

Sutherland, S. [2005]: «ePortfolios: a personal learning space», en S. Freitas de y C. Yapp, Personalisation in the 21st century, Stafford, Network Press.

Ward, R. y Grant, S. [2007]. What is an ePortfolio? Disponible en: http://www.recordingachievement.org/downloads/DrafteportfoliobriefingpaperSGRW13.pdf [Consulta: 15 de enero de 2015].

Watson, L. [2003]: Lifelong learning in Australia, Canberra, Department of Education, Science and Training (DEST).

Wenden, A. y Rubin, J. [1987]: Learner strategies in language learning, London, Prentice Hall.

Yancey, K. [2001]: «General patterns and the future», in B. Cambridge (ed.), Electronic portfolios: emerging practices in student, faculty, and institutional learning, Washington, DC, American Association for Higher Education, págs. 83-90.

Zimmerman, B. J. [2001]: «Achieving academic excellence: a self regulatory perspective», en M. Ferrari (ed.), The pursuit of excellence through education, Mahwah (NJ), Erlbaum, págs. 85-110. 
En www.cef.es/libros encontrará la totalidad de las publicaciones especializadas del EF.- y de la udima, con amplios sumarios para conocer los contenidos de cada una de las obras y su disponibilidad en papel, pdf o ePub.

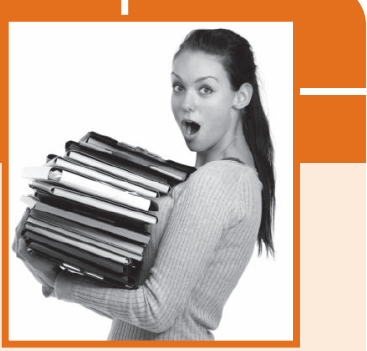

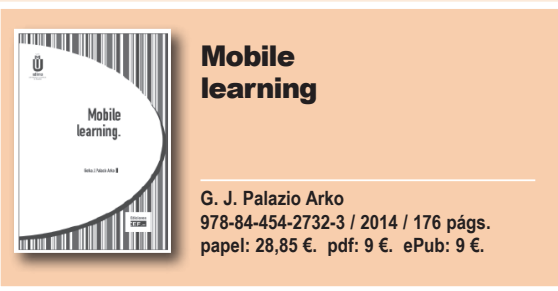
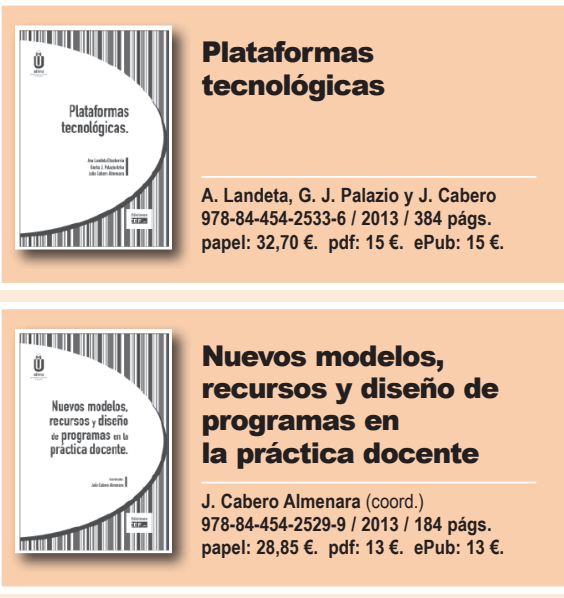

\begin{tabular}{|c|c|}
\hline 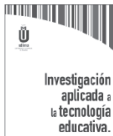 & $\begin{array}{l}\text { Investigación } \\
\text { aplicada a la } \\
\text { tecnología educativa }\end{array}$ \\
\hline$\|||||||||||||||||||$ & $\begin{array}{l}\text { J. Cabero Almenara } \\
\text { 978-84-454-2673-9 / } 2014 \text { / } 216 \text { págs. } \\
\text { papel: } 28,85 € . \text { pdf: } 15 € .\end{array}$ \\
\hline
\end{tabular}

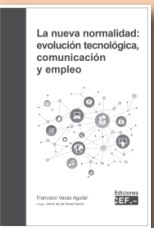

\section{La nueva normalidad: evolución tecnológica, comunicación y empleo}

F. Vacas Aguilar 978-84-454-2868-9 / 2014 / 152 págs. papel: $9,61 €$. pdf: $4,13 €$. ePub: $4,13 €$.
Prólogo. mLearning: concepto y uso. Hardware y sistemas operativos para el mLearning. Tiendas on-line con sus aplicaciones móviles de uso educativo. Sistemas, herramientas y plataformas para el mLearning (I). Sistemas, herramientas y plataformas para el mlearning (II). Diseño de aplicaciones y acciones formativas para el mLearning Índice sistemático

Sistemas de gestión de contenidos, características y funcionalidades de los gestores de contenidos. Sistemas de gestión de contenidos de código abierto, comerciales y sistemas de groupware. Sistemas de gestión orientados a la educación. Herramientas de las plataformas. Sindicación, agregación y curación de contenidos. Herramientas. Ventas y gestión de contenidos en soporte digital. Evaluación y análisis de las plataformas de teleformación. Enseñar y aprender en un entorno virtual basado en software libre. Moodle. Plataformas tecnológicas españolas y europeas. Índice sistemático

Presentación. El diseño instruccional: referencias iniciales. El diseño de la programación del aprendizaje en acciones de formación e-learning y b-learning. Estrategias y acciones metodológicas del tutor virtual. Diseño de e-actividades. Formación en competencias. Caso práctico sobre el desarrollo de una asignatura en ECTS. Bibliografía. Índice sistemático

Investigación cuantitativa y cualitativa en educación. Instrumentos de análisis y recogida de información en la investigación en tecnología educativa. Investigación y nuevos planteamientos didácticocurriculares. Diseño y producción de materiales educativos. Evaluación de medios y materiales de enseñanza. Aportaciones desde la investigación en tecnología educativa, para la aplicación educativa de Internet. Profesorado e integración escolar de los medios y las tecnologías de la información y la comunicación. Índice sistemático

Prólogo. Introducción. Digitalizando (la técnica y el impacto). La triple convergencia. El nuevo mercado de la movilidad. La crisis de los medios (de la mediación al consumo). Medios sociales. El (tortuoso) camino a la gratuidad. El cambio educativo (realidades de facto y apertura). El nuevo mercado laboral: de las corporaciones a las personas. La innovación permanente. Conclusiones (todo abierto, todo conectado). Fuentes consultadas y recomendadas. Glosario de términos. Glosario de siglas. Índice sistemático 